\title{
BOUNDARIES OF CYCLE SPACES AND DEGENERATING HODGE STRUCTURES*
}

\author{
TATSUKI HAYAMA ${ }^{\dagger}$
}

\begin{abstract}
We study a property of cycle spaces in connection with degenerating Hodge structures of odd-weight, and we construct maps from some partial compactifications of period domains to the Satake compatifications of Siegel spaces. These maps are a generalization of the maps from the toroidal compactifications of Siegel spaces to the Satake compactifications. We also show continuity of these maps for the case for the Hodge structure of Calabi-Yau threefolds with $h^{2,1}=1$.
\end{abstract} space.

Key words. Degenerating Hodge structure, partial compactification of period domain, cycle

AMS subject classifications. 32G20, $14 \mathrm{D} 07$.

1. Introduction. A cycle space is a space which parametrizes certain compact manifolds in a flag domain. This is studied in the field of complex geometry (cf. [FHW]). Our aim is to study degenerating Hodge structures by using the theory of cycle spaces. To be more specific, we will study the partial compactifications of period domains introduced by Kato and Usui $[\mathrm{KU}]$ in this paper. We will show some results on certain partial compactifications by using a property of cycle spaces. Furthermore, we will give an explicit calculation and a further result especially for the case for the Hodge structures of Calabi-Yau threefolds with $h^{2,1}=1$.

1.1. Period domains and the partial compactifications. By Griffiths $[G]$, a variation of Hodge structure gives a horizontal map to the period domain called a period map. Kato and Usui [KU] constructed partial compactifications so that period maps can be extended. They showed these partial compactifications are moduli spaces of degenerating Hodge structures called log Hodge structures. If we consider the Hodge structures of curves or K3 surfaces, period domains are Hermitian symmetric domains. For Hermitian symmetric domains, there are several ways to make compactifications. In particular, toroidal partial compactifications [AMRT] coincide with the partial compactifications of $[\mathrm{KU}]$. These partial compactifications are given by fans, and then the properties of fans have an effect on the properties of geometry on the partial compactifications. Ash, Mumford, Rapoport and Tai [AMRT] gave the constructions of fans which give compactifications.

For a general period domain, $[\mathrm{KU}]$ showed fundamental geometric properties of the partial compactifications of period domains using log geometry. The partial compactifications are not analytic spaces but analytic spaces with slits called log manifolds. In contrast with Hermitian symmetric case, the partial compactifications are not well-studied if period domains are not Hermitian symmetric. In fact, we do not have a general construction of fans in this situation.

In this paper, we will discuss certain partial compactifications of period domains of odd-weight Hodge structures. We will show some properties on these partial compactifications.

\footnotetext{
${ }^{*}$ Received September 3, 2012; accepted for publication June 14, 2013.

$\dagger$ Department of Mathematics, National Taiwan University, Taipei 106, Taiwan. Current address: Mathematical Sciences Center, Tsinghua University, Haidian District, Beijing 100084, China (tatsuki @math.tsinghua.edu.cn). Supported by National Science Council of Taiwan.
} 
1.2. Cycle spaces. We review cycle spaces briefly following [FHW]. Let $D$ be a period domain. Then $D$ is an open orbit of the flag manifold $\check{D}$ called the compact dual. Here the real Lie group $G$ act on $D$ transitively. Fixing a base point $F_{0}$ of $D$, the isotropy subgroup $L_{0}$ is compact, which is maximally compact if and only if $D \cong G / L_{0}$ is a Hermitian symmetric domain. Taking a maximally compact subgroup $K_{0}$ containing $L_{0}$, we have the orbit $C_{0}=K_{0} F_{0}$, which is a compact submanifold contained in $D$. The cycle space $\mathcal{M}_{D}$ is a set of all $g C_{0}$ with $g \in G_{\mathbb{C}}$ which is contained in $D$.

In this paper, we treat a case for odd-weight Hodge structures. By shifting, we may assume the weight is -1 . In this case we have

$$
G \cong S p(n, \mathbb{R}), \quad L_{0} \cong \prod_{j \geq 0} U\left(n_{j}\right), \quad K_{0} \cong U(n), \quad C_{0} \cong K_{0} / L_{0}
$$

where $\sum_{j} n_{j}=n$ and $\left\{n_{j}\right\}_{j}$ depends on the Hodge numbers (see [CMP]). Here $G / K_{0}$ is isomorphic to the Siegel space $\mathscr{H}$ of degree $n$, and we have the real analytic quotient map

$$
D \cong G / L_{0} \rightarrow G / K_{0} \cong \mathscr{H}
$$

Through this map, a $\mathbb{Z}$-Hodge structure whose Hodge filtration is in $D$ corresponds to an abelian variety, which coincide with the Weil intermediate Jacobian. Now $K_{0}=L_{0}$ if $h^{p,-p-1}=0$ for $p \neq 0,1$ (the case for Hodge structures of curves). Moreover $\mathcal{M}_{D} \cong \mathscr{H}$ if $K_{0}=L_{0}$.

We are mainly interested in the case where $L_{0} \neq K_{0}$, i.e. the case where $D$ is not a symmetric space. In this case the cycle space can be written by $\mathcal{M}_{D} \cong \mathscr{H} \times \overline{\mathscr{H}}$ where $\overline{\mathscr{H}}$ is its complex conjugate by [FHW]. Since $\mathscr{H}$ is a familiar object, $\mathcal{M}_{D}$ is easier to study than $D$ itself. In [H] we showed some properties of $\mathcal{M}_{D}$ in connection with $S L(2)$-orbits. In this paper we will give a generalization of the results of $[\mathrm{H}]$ in Proposition 2.4.

1.3. Degenerating Hodge structures. We may regard a nilpotent orbit as a degenerating Hodge structure by Schmid [S] and Cattani, Kaplan and Schmid [CKS]. A nilpotent orbit is given by a rational nilpotent cone in the Lie algebra of $G$ and an orbit in $\check{D}$ defined by this cone. Boundary points of the partial compactifications of period domains correspond to nilpotent orbits.

Let $\sigma$ be a nilpotent cone, and let $\mathbf{B}(\sigma)$ be the set of all $\sigma$-nilpotent orbits. For a fan $\Sigma$ of nilpotent cones, we denote by $D_{\Sigma}$ the union of $\mathbf{B}(\sigma)$ for $\sigma \in \Sigma$, where the component $\mathbf{B}(\{0\})$ for the 0 -cone is $D$. Taking a subgroup $\Gamma$ of the discrete group $G_{\mathbb{Z}}$ which is compatible with $\Sigma, \Gamma \backslash D_{\Sigma}$ is the partial compactification related to $\Sigma$. We will define an even-type (resp. odd-type) nilpotent cone $\sigma$ in Definition 2.6 and the Satake boundary component $\mathbf{B}_{S}(\sigma)$ of $\mathscr{H}$ corresponding to $\sigma$. By using a properties of cycle spaces (Proposition 2.4), we will construct a map

$$
p^{\mathrm{ev}}: \mathbf{B}(\sigma) \rightarrow \mathbf{B}_{S}(\sigma), \quad\left(\text { resp. } p^{\text {od }}: \mathbf{B}(\sigma) \rightarrow \overline{\mathbf{B}_{S}(\sigma)}\right) .
$$

If $D$ is a Siegel space, $p^{\text {ev }}$ coincides with the map $\zeta$ given by [CCK] (see Example $2.9)$.

THEOREM 1.1. Let $\Sigma$ be an even-type (resp. odd-type) fan (Definition 2.6), and let $\Gamma$ be a subgroup of $G_{\mathbb{Z}}$ which is compatible with $\Sigma$. Then we have the map $p^{\text {ev }}$ (resp.

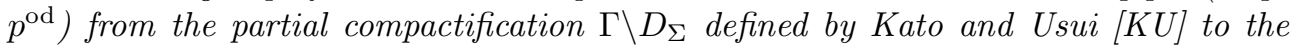




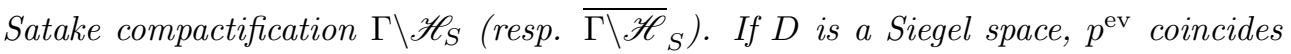
with the map from the toroidal partial compactification to the Satake compactification.

Moreover, an even-type (resp. odd-type) cone $\sigma$ gives a nilpotent orbit for $\mathscr{H}$ (resp. $\overline{\mathscr{H}})$. We denote by $\mathbf{B}_{\text {tor }}(\sigma)$ the boundary component of $\mathscr{H}_{\sigma}$. We will define the map

$$
\tilde{p}^{\mathrm{ev}}: \mathbf{B}(\sigma) \rightarrow \mathbf{B}_{\mathrm{tor}}(\sigma) \quad\left(\text { resp. } \tilde{p}^{\mathrm{od}}: \mathbf{B}(\sigma) \rightarrow \overline{\mathbf{B}_{\text {tor }}(\sigma)}\right)
$$

If $D$ is a Siegel space, $\tilde{p}^{\text {ev }}$ is the identity map. Then we will obtain the following theorem:

THEOREM 1.2. Let $\Sigma$ be an even-type (resp. odd-type) fan, and let $\Gamma$ be a subgroup of $G_{\mathbb{Z}}$ which is compatible with $\Sigma$. Then we have the map $\tilde{p}^{\text {ev }}: \Gamma \backslash D_{\Sigma} \rightarrow \Gamma \backslash \mathscr{H}_{\Sigma}$ (resp. $\tilde{p}^{\text {od }}: \Gamma \backslash D_{\Sigma} \rightarrow{\overline{\Gamma \backslash \mathscr{H}_{\Sigma}}}_{\Sigma}$ ), which factors through the map of Theorem 1.1. If $D$ is a Siegel space, $\tilde{p}^{\text {ev }}$ is the identity map.

Finally, we have the following commutative diagrams:
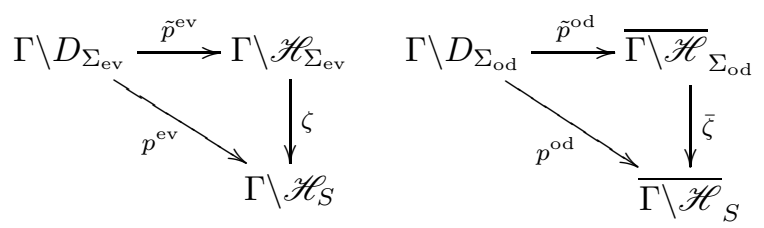

where $\Sigma_{\text {ev }}$ is an even-type fan and $\Sigma_{\text {od }}$ is an odd-type fan.

1.4. The $(\mathbf{1}, \mathbf{1}, \mathbf{1}, \mathbf{1})$ case. We will study these maps in detail for the case for the Hodge structures of Calabi-Yau threefolds with $h^{2,1}=1$. This case geometrically corresponds to the quintic mirror family or the Borcea-Voisin mirror family (see [GGK1]), and the nilpotent orbits are explicitly described and classified by Kato and Usui [KU]. We have the fans $\Sigma_{\mathrm{ev}}$ and $\Sigma_{\mathrm{od}}$ and the nilpotent orbits in this case. By using it, we will describe the maps $p^{\text {ev }}, p^{\text {od }}, \tilde{p}^{\text {ev }}$ and $\tilde{p}^{\text {od }}$ and show the following proposition:

Proposition 1.3. In this case, $\tilde{p}^{\text {ev }}$ and $\tilde{p}^{\text {od }}$ (therefore $p^{\mathrm{ev}}$ and $p^{\text {od }}$ ) are continuous.

Here the topology of $\Gamma \backslash D_{\Sigma}$ is the strong topology, which makes these maps continuous. Remark that the even-types and the odd-types are not parallel. Indeed, $p^{\text {od }}$ is not surjective although $p^{\mathrm{ev}}$ is, and $\Sigma_{\text {od }}$ is not a part of a fan of a toroidal compactification although $\Sigma_{\mathrm{ev}}$ is. In particular, the following diagram holds for the even-case (see Remark 3.4):

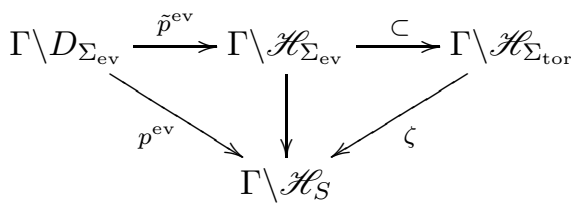

where $\Gamma \backslash \mathscr{H}_{\Sigma_{\text {tor }}}$ is the toroidal compactification. The even-case is further investigated in $[\mathrm{H} 2]$. 
1.5. Problems. How to construct $\Sigma_{\text {ev }}$ and $\Sigma_{\text {od }}$ and the property of $p^{\text {ev }}, p^{\text {od }}, \tilde{p}^{\text {ev }}$ and $\tilde{p}^{\text {od }}$ beyond the above case is unknown. We expect that $p^{\text {ev }}$ and $p^{\text {od }}$ have a good geometric property similar to the Siegel case. Since the toroidal compactifications and the Satake compactifications are well known, we expect that these maps are helpful to study the geometry of $\Gamma \backslash D_{\Sigma_{\text {ev }}}$ and $\Gamma \backslash D_{\Sigma_{\text {od }}}$.

On the other hand, Green, Griffiths and Kerr [GGK2] recently study MumfordTate domains. Kerr and Pearlstein $[\mathrm{KP}]$ investigate a relationship between boundary components of Mumford-Tate domains and the Kato-Usui boundary components. We expect that our study of Kato-Usui boundary components can be applied to the study of boundary components of Mumford-Tate domains.

Acknowledgment. The part of this work was done during a visit of the author to Johns Hopkins University for the activity of JAMI in February 2012. The author is grateful for the hospitality and the support. The author is thankful to professors Radu Laza, Gregory Pearlstein and Steven Zucker for their valuable advice and warm encouragement.

2. Even-type and odd-type Degenerations. Let $\left(H_{\mathbb{Z}}, F_{0},\langle\rangle,\right)$ be a polarized Hodge structure of weight -1 where $H_{\mathbb{Z}}$ is $\mathbb{Z}$-module, $F_{0}$ is a filtration of $H_{\mathbb{C}}:=H_{\mathbb{Z}} \otimes \mathbb{C}$ and $\langle$,$\rangle is a non-degenerate alternating bilinear form on H_{\mathbb{Z}}$. We have the period domain $D$ and its compact dual $\check{D}$ by [G]. Here $D$ is written as a homogeneous space on which the real Lie group $G$ acts. As in $(1.1), G \cong S p(n, \mathbb{R})$ and the isotropy subgroup $L_{0}$ is isomorphic to $\prod_{j \geq 0} U\left(n_{j}\right)$ where $n_{j}$ is the Hodge number $h^{j,-j-1}$. We denote by $\mathfrak{g}$ the Lie algebra of $G$.

2.1. $\mathbb{R}$-split LMHS with $N^{2}=0$. Let $N \in \mathfrak{g}$ be a nilpotent with $N^{2}=0$, and let $F \in \check{D}$. We have the monodromy weight filtration $W(N)$ such that

$$
W(N)_{0}=H_{\mathbb{R}}, \quad W(N)_{-1}=\operatorname{Ker} N, \quad W(N)_{-2}=\operatorname{Im} N
$$

(which is shifted by -1 from the original definition of the weight filtration). We say $(W(N), F)$ is a limiting mixed Hodge structure (LMHS) if the following properties hold:

- $(W(N), F)$ is a mixed Hodge structure;

- $N: \mathrm{Gr}_{0}^{W(N)} \stackrel{\sim}{\rightarrow} \mathrm{Gr}_{-2}^{W(N)}$ gives a $(-1,-1)$-morphism of Hodge structure;

- $\left\langle\bullet, N^{j} \bullet\right\rangle$ gives a polarization for $\mathrm{Gr}_{j-1}^{W(N)}(j=0,1)$.

Now we have the Deligne decomposition $H_{\mathbb{C}}=\bigoplus_{p+q=0,-1,-2} I^{p, q}$ letting

$$
I^{p, q}=F^{p} \cap W(N)_{p+q, \mathbb{C}} \cap\left(\bar{F}^{q} \cap W(N)_{p+q, \mathbb{C}}+\sum_{j \geq 2} \bar{F}^{q-j+1} \cap W(N)_{p+q-j, \mathbb{C}}\right),
$$

which gives Hodge decomposition on each graded quotient part. We assume that the LMHS is $\mathbb{R}$-split, i.e. the Deligne decomposition is defined over $\mathbb{R}$. By [CKS, Lemma 3.12], $\exp (z N) F \in D$ for $\operatorname{Im} z>0$.

Lemma 2.1. Let $v \in I^{p,-p}$. For $z \in \mathbb{C}$ with $\operatorname{Im} z>0$, $\exp (z N) v$ is in the $(p,-p-1)$-component of the Hodge decomposition with respect to $\exp (z N) F$.

Proof. Now $\exp (z N) v \in \exp (z N) F^{p}$. Moreover, $\bar{v} \in F^{-p}$ and $N \bar{v} \in F^{-p-1}$ since the LMHS is $\mathbb{R}$-split. Here

$$
\begin{aligned}
& N \bar{v}=\exp (z N)(N \bar{v}) \in \exp (z N) F^{-p-1} \\
& \bar{v}-z N \bar{v}=\exp (z N) \bar{v}-2 z N \bar{v} \in \exp (z N) F^{-p-1} .
\end{aligned}
$$


Then

$$
\begin{aligned}
\exp (z N) v & =v+z N v \\
& =\overline{\bar{v}-z N \bar{v}}+2 \operatorname{Re}(z) N v \in \overline{\exp (z N) F^{-p-1}}
\end{aligned}
$$

Let $H_{\mathbb{C}}=\bigoplus_{p} H^{p,-p-1}$ be the Hodge decomposition with respect to $\exp (z N) F$ for $\operatorname{Im} z>0$. By the above lemma,

$$
\begin{aligned}
& e^{z N} I^{p,-p} \subset H^{p,-p-1}, \quad e^{z N} I^{-p, p} \subset H^{-p, p-1}, \\
& e^{\bar{z} N} I^{-p, p}=\overline{e^{z N} I^{p,-p}} \subset H^{-p-1, p}, \quad e^{\bar{z} N} I^{p,-p}=\overline{e^{z N} I^{-p, p}} \subset H^{p-1,-p} .
\end{aligned}
$$

We write

$$
H_{1}^{p,-p-1}=I^{p,-p-1}, \quad H_{2}^{p,-p-1}=e^{z N} I^{p,-p}, \quad H_{3}^{p,-p-1}=e^{\bar{z} N} I^{p+1,-p-1} .
$$

Then the $(p,-p-1)$-component has the decomposition

$$
H^{p,-p-1}=H_{1}^{p,-p-1} \oplus H_{2}^{p,-p-1} \oplus H_{3}^{p,-p-1} .
$$

REMARK 2.2. By [S, Lemma 6.24], $H_{\mathbb{C}}$ can be decomposed into the direct sum of the subspaces which are invariant and irreducible with respect to the Hodge structure. Here every irreducible subspace is isomorphic to $H(p) \otimes S(-2 p-1)$ with $p \leq-1$, or $E(p, q) \otimes S(-p-q-1)$ with $p+q \leq-1$. The relationship between this decomposition and the decomposition (2.2) is written as follows:

$$
H_{1}^{p,-p-1} \oplus H_{1}^{-p-1, p}
$$

is the direct sum of the $(E(p,-p-1) \otimes S(0))$ type components;

$$
\begin{aligned}
& H_{2}^{p,-p-1} \oplus H_{3}^{p-1,-p} \oplus H_{2}^{-p, p-1} \oplus H_{3}^{-p-1, p} \\
& =I^{p,-p} \oplus I^{-p, p} \oplus I^{p-1,-p-1} \oplus I^{-p-1, p-1}
\end{aligned}
$$

is the direct sum of the $(E(p-1,-p-1) \oplus S(1))$ type components for $p \geq 1$;

$$
H_{2}^{0,-1} \oplus H_{3}^{-1,0}=I^{0,0} \oplus I^{-1,-1}
$$

is the direct sum of the $(H(-1) \oplus S(1))$ type components.

2.2. Cycle spaces and $\mathbf{S L}(2)$-orbits. Let $(N, F)$ be a pair which generates a LMHS with $N^{2}=0$. By [CKS, Proposition 2.20], there exists $\delta \in L_{\mathbb{R}}^{-1,-1}(W(\sigma), F)$ uniquely such that $\left(W(\sigma), e^{-i \delta} F\right)$ is a $\mathbb{R}$-split LMHS. We write $\hat{F}=e^{-i \delta} F$. By the SL(2)-orbit theorem ([S, Theorem 5.13], [CKS, §3]), there exists a Lie group homomorphism $\rho: S L(2, \mathbb{C}) \rightarrow G_{\mathbb{C}}$ defined over $\mathbb{R}$ and a holomorphic map $\phi: \mathbb{P}^{1} \rightarrow \check{D}$ satisfying the following conditions:

- $\rho(g) \phi(z)=\phi(g z)$;

- $\phi(0)=\hat{F}$

- $\rho_{*}\left(\mathbf{n}_{-}\right)=N$;

- $H v=(p+q+1) v$ for $v \in I^{p, q}$ where $\rho_{*}(\mathbf{h})=H$; 
- $\rho_{*}: \mathfrak{s l}(2, \mathbb{C}) \rightarrow \mathfrak{g}_{\mathbb{C}}$ is a $(0,0)$-morphism of Hodge structure where $\mathfrak{g}$ (resp. $\mathfrak{s l}(2, \mathbb{R}))$ has a Hodge structure of weight 0 relative to $\phi(i)$ (resp. $i)$,

where $\left\{\mathbf{n}_{-}, \mathbf{h}, \mathbf{n}_{+}\right\}$are the standard generators of $s l(2, \mathbb{C})$. We fix $F_{0}=\phi(i)=$ $\exp (i N) \hat{F}$ as a base point of $D$. We write

$$
X=\frac{1}{2}\left(i N-H+i N^{+}\right)
$$

where $N^{+}=\rho_{*}\left(\mathbf{n}_{+}\right)$. Then $X$ is in the $(-1,1)$-component of the Hodge decomposition of $\mathfrak{g}_{\mathbb{C}}$ with respect to $F_{0}$ and $X^{2}=0$. Let $H_{\mathbb{C}}=\bigoplus H^{p,-p-1}$ be the Hodge decomposition with respect to $F_{0}$. By Lemma 2.1, for $v \in I^{p,-p}$ we have

$$
u=\exp (i N) v \in H^{p,-p-1}
$$

Then $X u \in H^{p-1,-p}$. We denote by $\|\bullet\|$ the norm induced by the polarization with respect to $F_{0}$. Scaling $v$, we may assume $\|u\|=1$.

Lemma 2.3. $X u=-\exp (-i N) v$ and $\|X u\|=1$.

Proof. By the property of the $s l_{2}$-triple,

$$
\begin{aligned}
& N^{+} N v=v, \quad N^{+} N \bar{v}=\bar{v}, \quad N^{+} v=N^{+} \bar{v}=0, \\
& H v=v, \quad H \bar{v}=\bar{v}, \quad H N v=-N v, \quad H N \bar{v}=-N \bar{v} .
\end{aligned}
$$

Then

$$
\begin{aligned}
X u & =\frac{1}{2}\left(i N-H+i N^{+}\right)(v+i N v) \\
& =-v+i N v=-\exp (-i N) v .
\end{aligned}
$$

Next, we show $\|X u\|=1$. Let $a=\langle v, \bar{v}\rangle, b=\langle N v, \bar{v}\rangle, c=\langle v, N \bar{v}\rangle$ and $d=$ $\langle N v, N \bar{v}\rangle$. Then by the orthogonality and the positivity

$$
\begin{aligned}
& \langle u, \bar{u}\rangle=a+i b-i c+d=i^{-2 p-1}, \quad\langle u, \overline{X u}\rangle=-a-i b-i c+d=0, \\
& \langle X u, \bar{u}\rangle=-a+i b+i c+d=0 .
\end{aligned}
$$

Since $v \in \hat{F}^{p}$ and $\bar{v} \in \hat{F}^{-p}, a=0$. Therefore, the simultaneous equation induces $d=0, b-c=i^{-2 p-2}$ and $\langle X u, \overline{X u}\rangle=a-i b+i c+d=-i^{-2 p-1}$. Then $\|X u\|=$ $i^{2 p-1}\langle X u, \overline{X u}\rangle=1$.

Then $X$ gives the isomorphism

$$
X: H_{2}^{p,-p-1} \rightarrow H_{3}^{p-1,-p} ; \quad \exp (i N) v \mapsto-\exp (-i N) v .
$$

Therefore, we have

$$
i^{2 p+1}\langle\exp (z X) u, \overline{\exp (z X) u}\rangle=1-|z|^{2} .
$$

If $v^{\prime} \in I^{p,-p}$ is orthogonal to $v$ for $\langle\bullet, N \bar{\bullet}\rangle$,

$$
\left\langle\exp (z X) u, \overline{\exp (z X) u^{\prime}}\right\rangle=0
$$

where $u^{\prime}=\exp (i N) v^{\prime}$. 
For the Hodge numbers $\left\{h^{p,-p-1}\right\}_{p}$, we define

$$
f_{\mathrm{ev}}^{p}=\sum_{\substack{r \geq p, r: \text { even }}} h^{r,-r-1}, \quad f_{\text {od }}^{p}=\sum_{\substack{r \geq p, r: \text { odd }}} h^{r,-r-1} .
$$

The maximally compact subgroup $K_{0}$ is isomorphic to the unitary group $U(n)$ as in (1.1), and the orbit $C_{0}=K_{0} F_{0}$ is a compact submanifold of $D$ by [FHW, Lemma 5.1.3]. The cycle space is defined by

$$
\mathcal{M}_{D}=\left\{g C_{0} \mid g C_{0} \subset D, g \in G_{\mathbb{C}}\right\} .
$$

By [FHW, Lemma 5.1.3], $\mathcal{M}_{D}$ is an open subset of the complex manifold

$$
\mathcal{M}_{\check{D}}=\left\{g C_{0} \mid g \in G_{\mathbb{C}}\right\} .
$$

If $K_{0}=L_{0}$, i.e. $D$ is a Siegel space, $C_{0}$ is the base point $F_{0}$ and $\mathcal{M}_{D}=D$. We describe $\mathcal{M}_{D}$ for the case where $K_{0} \neq L_{0}$ following [FHW, $§ 5.5 \mathrm{~B}$ ]. Here $C_{0}$ can be written as

$$
C_{0}=\left\{F \in D \mid \operatorname{dim}\left(F^{p} \cap H^{\mathrm{ev}}\right)=f_{\mathrm{ev}}^{p}, \operatorname{dim}\left(F^{p} \cap H^{\mathrm{od}}\right)=f_{\mathrm{od}}^{p}\right\}
$$

where

$$
H^{\mathrm{ev}}=\bigoplus_{p: \text { even }} H^{p, q}, \quad H^{\mathrm{od}}=\bigoplus_{p: \text { odd }} H^{p, q}
$$

Let $V$ and $W$ be complementary $\langle$,$\rangle -isotropic n$-dimensional subspaces of $H_{\mathbb{C}}$, and let

$$
C_{V, W}=\left\{F \in \check{D} \mid \operatorname{dim}\left(F^{p} \cap V\right)=f_{\mathrm{ev}}^{p}, \operatorname{dim}\left(F^{p} \cap W\right)=f_{\mathrm{od}}^{p}\right\} .
$$

Here $C_{0}=C_{H^{\mathrm{ev}}, H^{\text {od }}}$ and $g C_{V, W}=C_{g V, g W}$ for $g \in G_{\mathbb{C}}$. By using this, the cycle space is described as

$$
\mathcal{M}_{D}=\left\{C_{V, W} \mid V \gg 0 \text { and } W \ll 0 \text { for }-i\langle\bullet, \bar{\bullet}\rangle\right\} .
$$

Now $G / K_{0}$ is isomorphic to the Siegel space $\mathscr{H}$ of degree $n$. Moreover $\mathscr{H}$ is isomorphic to the bounded symmetric domain $\mathcal{B}$ of type-III. Then we have

$$
\begin{aligned}
& \left\{V \subset H_{\mathbb{C}} \mid \operatorname{dim} V=n, V \gg 0,\langle V, V\rangle=0\right\} \cong \mathscr{H} \cong \mathcal{B}, \\
& \left\{W \subset H_{\mathbb{C}} \mid \operatorname{dim} W=n, W \ll 0,\langle W, W\rangle=0\right\} \cong \overline{\mathscr{H}} \cong \overline{\mathcal{B}} .
\end{aligned}
$$

By [FHW, §5.4], we have the isomorphism

$$
\mathcal{M}_{D} \stackrel{\sim}{\rightarrow} \mathcal{B} \times \overline{\mathcal{B}} ; \quad C_{(V, W)} \mapsto(V, W) .
$$

Proposition 2.4. $\exp (X) C_{0} \in \mathcal{M}_{\check{D}}$ is in the topological closure $\mathcal{M}_{D}^{\mathrm{cl}}$ of $\mathcal{M}_{D}$ in $\mathcal{M}_{\check{D}}$ if $N^{2}=0$.

Proof. Now we have the decomposition $H^{p,-p-1}=\bigoplus_{j=1,2,3} H_{j}^{p,-p-1}$ of $(2.2)$ with respect to $\exp (i N) \hat{F}$. Since $X H_{1}^{p,-p-1}=X H_{3}^{p,-p-1}=0$, then

$$
e^{X} H^{p, p-1}=H_{1}^{p, p-1} \oplus e^{X} H_{2}^{p, p-1} \oplus H_{3}^{p, p-1} .
$$

Here the above components are orthogonal to each other, and by (2.3) and (2.4) $e^{X} H_{2}^{p, p-1}$ is non-negative (resp. non-positive) for $i^{2 p-1}\langle\bullet, \bar{\bullet}\rangle$ if $p$ is even (resp. odd). Then $e^{X} H^{\text {ev }}$ (resp. $\left.e^{X} H^{\text {od }}\right)$ is in the closure $\mathcal{B}^{\text {cl }}\left(\right.$ resp. $\left.\overline{\mathcal{B}}^{\text {cl }}\right)$.

REMARK 2.5. If $N^{2} \neq 0, e^{X} C_{0}$ need not to be in $\mathcal{M}_{D}^{\text {cl }}$ (See [H, proposition 4.6]). 
2.3. Maps to the Satake boundary components. We call $\left(\sigma, \exp \left(\sigma_{\mathbb{C}}\right) F\right)$ a nilpotent orbit if it satisfies the following conditions:

- $\sigma$ is a finitely generated rational polyhedral cone in $\mathfrak{g}$;

- Any elements of $\sigma$ are nilpotent and commutative with each other;

- $F \in \check{D}$ and $\exp (z N) F \in D$ for $\operatorname{Im} z \gg 0$ and for $N$ in the relative interior $\sigma^{\circ}$;

- $N F^{p} \subset F^{p-1}$ for $N \in \sigma$.

The above conditions do not depend on the choice of $F \in \exp \left(\sigma_{\mathbb{C}}\right) F$. By $[\mathrm{CK}]$ the monodromy weight filtration $W(N)$ does not depend on the choice of $N \in \sigma^{\circ}$ (we denote it by $W(\sigma))$, and $(W(\sigma), F)$ is a LMHS by [S].

Definition 2.6. A nilpotent orbit $\left(\sigma, \exp \left(\sigma_{\mathbb{C}}\right) F\right)$ is called even-type (resp. oddtype) if $N^{2}=0$ for $N$ in the relative interior $\sigma^{\circ}$ and $I^{p,-p}=0$ for any odd (resp. even) integer $p$ with respect to the LMHS $(W(\sigma), F)$. A nilpotent cone $\sigma$ is called even-type (resp. odd-type) if every $\sigma$-nilpotent orbit is even-type (resp. odd-type). A fan $\Sigma$ is called even-type (resp. odd-type) if any face of $\Sigma$ is even-type (resp. odd-type).

Let $\left(\sigma, \exp \left(\sigma_{\mathbb{C}}\right) F\right)$ be a nilpotent orbit of even-type or odd-type. For $(N, F)$ with $N \in \sigma^{\circ}$, we have the compact submanifold $C_{0} \in \mathcal{M}_{D}$ as in the previous subsection. By Proposition 2.4, $\exp (X) C_{0} \in \mathcal{M}_{D}^{\text {cl }}$. If $K_{0} \neq L_{0}, \mathcal{M}_{D} \cong \mathcal{B} \times \overline{\mathcal{B}}$. We then define the two projections

$$
p^{\mathrm{ev}}: \mathcal{M}_{D}^{\mathrm{cl}} \rightarrow \mathcal{B}^{\mathrm{cl}}, \quad p^{\mathrm{od}}: \mathcal{M}_{D}^{\mathrm{cl}} \rightarrow \overline{\mathcal{B}}^{\mathrm{cl}}
$$

If $K_{0}=L_{0}$, we define $p^{\text {ev }}$ as the canonical isomorphism $\mathcal{M}_{D}^{\text {cl }} \stackrel{\sim}{\rightarrow} \mathcal{B}^{\text {cl }}$ and $p^{\text {od }}$ as the complex conjugation map $\mathcal{M}_{D}^{\text {cl }} \stackrel{\sim}{\rightarrow} \mathcal{B}^{\text {cl }} \rightarrow \overline{\mathcal{B}}^{\text {cl }}$.

THEOREM 2.7. If the nilpotent orbit is of even-type (resp. odd-type), $p^{\mathrm{ev}}\left(\exp (X) C_{0}\right) \in \mathcal{B}^{\mathrm{cl}}\left(\right.$ resp. $\left.p^{\mathrm{od}}\left(\exp (X) C_{0}\right) \in \overline{\mathcal{B}}^{\mathrm{cl}}\right)$ does not depend on the choice of $N \in \sigma^{\circ}$ and $F \in \exp \left(\sigma_{\mathbb{C}}\right) F$.

Proof. By Lemma 2.1, $u=\exp (i N) v \in H_{2}^{p,-p-1}$ for $v \in I^{p,-p}$. By Lemma 2.3

$$
\exp (X) u=\exp (i N) v-\exp (-i N) v=2 i N v \in e^{X} H_{2}^{p,-p-1},
$$

and for $u^{\prime}=\exp (i N) \bar{v} \in H_{2}^{-p, p-1}$

$$
\exp (X) u^{\prime}=\exp (i N) \bar{v}-\exp (-i N) \bar{v}=2 i N \bar{v} \in e^{X} H_{2}^{-p, p-1} .
$$

Since $H_{3}^{p,-p-1}=0$ for even (resp. odd) $p$ by definition, then

$$
\begin{aligned}
& e^{X} H^{\text {ev }}=\bigoplus_{p: \text { even }}\left(H_{1}^{p, p-1} \oplus e^{X} H_{2}^{p, p-1}\right)=\bigoplus_{p \text { : even }} H_{1}^{p, p-1} \oplus \operatorname{Im} N_{\mathbb{C}} \\
& \text { (resp. } \left.e^{X} H^{\text {od }}=\bigoplus_{p \text { : odd }} H_{1}^{p, p-1} \oplus \operatorname{Im} N_{\mathbb{C}}\right) .
\end{aligned}
$$

Here $\operatorname{Im} N=W(\sigma)_{-2}$ does not depend on the choice of $N \in \sigma^{\circ}$. Moreover $H_{1}^{p,-p-1}$ is in the kernel of the action of $\sigma_{\mathbb{C}}$ and $L_{\mathbb{R}}^{-1,-1}(W(\sigma), F)$. Then $e^{X} H^{\text {ev }}\left(\right.$ resp. $\left.e^{X} H^{\text {od }}\right)$ does not depend on the choice of $F \in \exp \left(\sigma_{\mathbb{C}}\right) F$ and $N \in \sigma^{\circ}$.

The Satake boundary components of $\mathcal{B}$ corresponds to the set of real isotropic subspaces of $H_{\mathbb{R}}$ ([N, Proposition 4.4]). We denote by $\mathbf{B}_{S}(\sigma)$ the Satake boundary component corresponding to the real vector space $W(\sigma)_{-2}$. The boundary point 
$p^{\text {ev }}\left(\exp (X) C_{0}\right)$ (resp. $\left.p^{\text {od }}\left(\exp (X) C_{0}\right)\right)$ is contained in the Satake boundary component $\mathbf{B}_{S}(\sigma)\left(\operatorname{resp} . \overline{\mathbf{B}_{S}(\sigma)}\right)$.

COROllary 2.8. Let $\sigma$ be an even-type (resp. odd-type) nilpotent cone, and let $\mathbf{B}(\sigma)$ be the set of all $\sigma$-nilpotent orbits. Then $p^{\text {ev }}$ (resp. $p^{\text {od }}$ ) gives a well-defined $\operatorname{map} \mathbf{B}(\sigma) \rightarrow \mathbf{B}_{S}(\sigma)$ (resp. $\left.\mathbf{B}(\sigma) \rightarrow \overline{\mathbf{B}_{S}(\sigma)}\right)$.

Let $\Sigma$ be an even-type (resp. odd-type) fan. We write $D_{\Sigma}=\bigsqcup_{\sigma \in \Sigma} \mathbf{B}(\sigma)$. We have the well-defined map

$$
p^{\text {ev }}: D_{\Sigma} \rightarrow \mathscr{H}_{S} \quad\left(\text { resp. } p^{\text {od }}: D_{\Sigma} \rightarrow \overline{\mathscr{H}}_{S}\right)
$$

where the map restricted on $\mathbf{B}(\sigma)$ is given by Corollary 2.8. If $\sigma=\{0\}$, then $\mathbf{B}(\sigma)=D$ and $\left.p^{\text {ev }}\right|_{D}$ (resp. $\left.p^{\text {od }}\right|_{D}$ ) is the map given by taking the even-part (resp. odd-part) of the Hodge decomposition. Let $\Gamma$ be a subgroup of $G_{\mathbb{Z}}=\operatorname{Aut}\left(H_{\mathbb{Z}},\langle\rangle,\right)$. Then $\gamma \in \Gamma$ gives a map from $\mathbf{B}(\sigma)$ to $\mathbf{B}(\operatorname{Ad}(\gamma) \sigma)$ by

$$
\left(\sigma, \exp \left(\sigma_{\mathbb{C}}\right) F\right) \mapsto\left(\operatorname{Ad}(\gamma) \sigma, \gamma \exp \left(\sigma_{\mathbb{C}}\right) F\right)
$$

We assume that $\Gamma$ is compatible with the fan $\Sigma$. Then $p^{\text {ev }}$ (resp. $p^{\text {od }}$ ) is compatible with the action of $\Gamma$, and we can define

$$
p^{\text {ev }}: \Gamma \backslash D_{\Sigma} \rightarrow \Gamma \backslash \mathscr{H}_{S} \quad\left(\text { resp. } p^{\text {od }}: \Gamma \backslash D_{\Sigma} \rightarrow \overline{\Gamma \backslash \mathscr{H}_{S}}\right) .
$$

ExAmple 2.9. Let $D$ be a Siegel space $\mathscr{H}$, i.e. $h^{p,-p-1}=0$ if $p \neq 0,-1$. We take a nilpotent cone $\sigma$ in the open real cone $\eta_{i}^{+}$of [CCK, §4]. By [CCK, Proposition 4.2], $(W(\sigma), F)$ is a LMHS if and only if $F \in \exp \left(\sigma_{\mathbb{C}}\right) \mathscr{H} \subset \mathscr{\mathscr { H }}$, and

$$
\mathbf{B}(\sigma) \cong \exp \left(\sigma_{\mathbb{C}}\right) \mathscr{H} / \exp \left(\sigma_{\mathbb{C}}\right) .
$$

Here the LMHS is the following type:

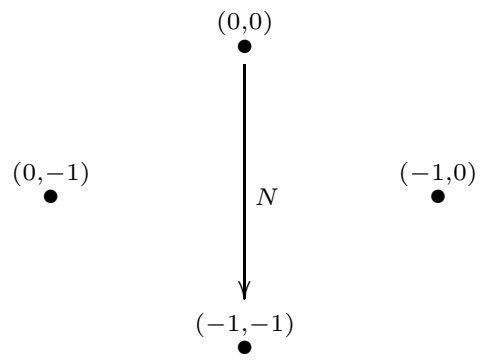

Then any nilpotent orbit for $\mathscr{H}$ is even-type, where $\left.p^{\mathrm{ev}}\right|_{D}=i d$ and $p^{\text {ev }}: \mathbf{B}(\sigma) \rightarrow$ $\mathbf{B}_{S}(\sigma)$ coincides with the map $\zeta$ of $[\mathrm{CCK}, \S 4(3)]$. By [CCK, $\left.\S 6\right] \zeta$ induces the maps from the toroidal compactifications to the Satake compactification.

2.4. Maps to the toroidal boundary components. For an even-type (resp. odd-type) nilpotent orbit $\left(\sigma, \exp \left(\sigma_{\mathbb{C}}\right) F\right)$, we have the $\mathbb{R}$-split nilpotent orbit $\left(\sigma, \exp \left(\sigma_{\mathbb{C}}\right) \hat{F}\right)$. Let $H_{\mathbb{C}}=\bigoplus_{p+q=0,-1,-2} I^{p, q}$ be the Deligne decomposition with respect to the LMHS $(W(\sigma), \hat{F})$. We define $\tilde{F} \in \check{\mathscr{H}}$ by

$$
\begin{aligned}
& \tilde{F}^{0}=\left(\bigoplus_{p: \text { even }} I^{p,-p-1}\right) \oplus\left(\bigoplus_{p} I^{p,-p}\right), \\
& \text { (resp. } \left.\tilde{F}^{0}=\left(\bigoplus_{p: \text { odd }} I^{p,-p-1}\right) \oplus\left(\bigoplus_{p} I^{p,-p}\right)\right) .
\end{aligned}
$$


Now $(W(\sigma), \tilde{F})$ (resp. $(W(-\sigma), \overline{\tilde{F}}))$ is a $\mathbb{R}$-split LMHS. In fact, $\langle\bullet, N \bullet\rangle$ (resp. $\langle\bullet,-N \bullet\rangle)$ with $N \in \sigma^{\circ}$ gives a polarization on $\mathrm{Gr}_{0}^{W(\sigma)}$ since $i^{2 p}=1$ if $p$ is even and -1 if $p$ is odd. Then $\left(\sigma, \exp \left(\sigma_{\mathbb{C}}\right) \tilde{F}\right)$ is a $\mathbb{R}$-split nilpotent orbit for $\mathscr{H}$ (resp. $\overline{\mathscr{H}}$ ). Moreover we have the following proposition:

Proposition 2.10. $p^{\text {ev }}\left(e^{z N} \hat{F}\right)=e^{z N} \tilde{F}$ (resp. $\left.p^{\text {od }}\left(e^{\bar{z} N} \hat{F}\right)=e^{\bar{z} N} \tilde{F}\right)$ for $\operatorname{Im} z>0$ and $N \in \sigma^{\circ}$.

Proof. Let $H_{\mathbb{C}}=\bigoplus_{p} H^{p,-p-1}$ be the Hodge decomposition with respect to $\exp (z N) \hat{F}$. Since $\sigma$ is even-type (resp. odd-type), $H_{3}^{p,-p-1}=0$ if $p$ is even (resp. odd). Then by (2.1)

$$
\begin{aligned}
p^{\mathrm{ev}}\left(e^{z N} \hat{F}\right)^{0} & =\bigoplus_{p: \text { even }}\left(H_{1}^{p,-p-1} \oplus H_{2}^{p,-p-1}\right) \\
& =\bigoplus_{p: \text { even }} I^{p,-p-1} \oplus e^{z N}\left(\bigoplus_{p} I^{p,-p}\right)=e^{z N} \tilde{F}^{0}
\end{aligned}
$$

(resp. $\left.p^{\text {od }}\left(e^{\bar{z} N} \hat{F}\right)^{0}=e^{\bar{z} N} \tilde{F}^{0}\right)$.

We denote by $\mathbf{B}_{\text {tor }}(\sigma)$ the boundary component for $\sigma$ of $\mathscr{H}_{\sigma}$. For an even-type (resp. odd-type) nilpotent cone $\sigma$, we define the map $\tilde{p}^{\text {ev }}: \mathbf{B}(\sigma) \rightarrow \mathbf{B}_{\text {tor }}(\sigma)$ (resp. $\left.\tilde{p}^{\text {od }}: \mathbf{B}(\sigma) \rightarrow \overline{\mathbf{B}_{\text {tor }}(\sigma)}\right)$ by

$$
\left(\sigma, \exp \left(\sigma_{\mathbb{C}}\right) F\right) \mapsto\left(\sigma, \exp \left(\sigma_{\mathbb{C}}\right) e^{i \delta} \tilde{F}\right)
$$

where $F=e^{i \delta} \hat{F}$. Then for an even-type (resp. odd-type) fan $\Sigma$ we can define the map

$$
\tilde{p}^{\text {ev }}: D_{\Sigma} \rightarrow \mathscr{H}_{\Sigma} \quad\left(\text { resp. } \tilde{p}^{\text {od }}: D_{\Sigma} \rightarrow \overline{\mathscr{H}}_{\Sigma}\right)
$$

and for a subgroup $\Gamma$ of $G_{\mathbb{Z}}$ which is compatible with $\Sigma$ we have

$$
\tilde{p}^{\text {ev }}: \Gamma \backslash D_{\Sigma} \rightarrow \Gamma \backslash \mathscr{H}_{\Sigma} \quad\left(\text { resp. } \tilde{p}^{\text {od }}: \Gamma \backslash D_{\Sigma} \rightarrow{\overline{\Gamma \backslash \mathscr{H}_{\Sigma}}}\right),
$$

where $\tilde{p}^{\text {ev }}=i d$ if $D=\mathscr{H}$. Now we have the map $\zeta: \Gamma \backslash \mathscr{H}_{\Sigma} \rightarrow \Gamma \backslash \mathscr{H}_{S}$ (resp. $\bar{\zeta}: \Gamma \backslash \mathscr{H}_{\Sigma} \rightarrow{\overline{\Gamma \backslash \mathscr{H}_{S}}}_{S}$ such that

$$
\begin{aligned}
\zeta \circ \tilde{p}^{\operatorname{ev}}\left(\sigma, \exp \left(\sigma_{\mathbb{C}}\right) F\right) & =W(\sigma)_{-2} \oplus\left(\bigoplus_{p: \text { even }} I^{p,-p-1}\right) \\
& =p^{\text {ev }}\left(\sigma, \exp \left(\sigma_{\mathbb{C}}\right) F\right)
\end{aligned}
$$

$\left(\right.$ resp. $\left.\bar{\zeta} \circ \tilde{p}^{\text {od }}\left(\sigma, \exp \left(\sigma_{\mathbb{C}}\right) F\right)=p^{\text {od }}\left(\sigma, \exp \left(\sigma_{\mathbb{C}}\right) F\right)\right)$. Then we have the following theorem:

ThEOREM 2.11. $p^{\text {ev }}$ (resp. $p^{\text {od }}$ ) factors through $\tilde{p}^{\text {ev }}$ (resp. $\left.\tilde{p}^{\text {od }}\right)$.

3. The $(\mathbf{1}, \mathbf{1}, \mathbf{1}, \mathbf{1})$ case. In this section, we consider the case where $h^{p,-p-1}=1$ if $p=1,0,-1,-2, h^{p,-p-1}=0$ otherwise. In this case

$$
G \cong S p(2, \mathbb{R}), \quad L_{0} \cong U(1) \times U(1), \quad K_{0} \cong U(2), \quad C_{0} \cong \mathbb{P}^{1} .
$$

Any nilpotent cone in this case is rank 1, and its generator $N$ is classified as follows:

(I) $N^{2}=0, \operatorname{dim}(\operatorname{Im} N)=1$; 
(II) $N^{2}=0, \operatorname{dim}(\operatorname{Im} N)=2$;

(III) $N^{3} \neq 0, N^{4}=0$.

Here the type I is even-type; the type II is odd-type; the type III is neither. The boundary components are described by [KU, §12.3] or [GGK1]. For the type I (resp. the type II), we describe $p^{\text {ev }}$ and $\tilde{p}^{\text {ev }}$ (resp. $p^{\text {od }}$ and $\tilde{p}^{\text {od }}$ ) explicitly and show continuity of these maps.

3.1. Preliminary. First, we describe the period domain $D$. Let $H_{\mathbb{Z}}$ be a rank-4 $\mathbb{Z}$-module. We define a bilinear form $\langle$,$\rangle by$

$$
\left(\left\langle e_{j}, e_{k}\right\rangle\right)_{j, k}=\left(\begin{array}{cc}
0 & -I \\
I & 0
\end{array}\right)
$$

for a basis $e_{1}, \ldots, e_{4}$ of $H_{\mathbb{Z}}$. We have an open immersion

$$
\begin{aligned}
& \operatorname{Sym}(2, \mathbb{C}) \times \mathbb{C} \hookrightarrow \check{D} ; \quad(\tau, \lambda) \mapsto F(\tau, \lambda) \text { where } \\
& F^{1}(\tau, \lambda)=\operatorname{span}_{\mathbb{C}}\left\{\left(\begin{array}{c}
\tau_{12} \\
\tau_{22} \\
0 \\
1
\end{array}\right)+\lambda\left(\begin{array}{c}
\tau_{11} \\
\tau_{21} \\
1 \\
0
\end{array}\right)\right\}, \quad F^{0}(\tau, \lambda)=\operatorname{span}_{\mathbb{C}}\left\{\left(\begin{array}{c}
\tau_{12} \\
\tau_{22} \\
0 \\
1
\end{array}\right),\left(\begin{array}{c}
\tau_{11} \\
\tau_{21} \\
1 \\
0
\end{array}\right)\right\}, \\
& F^{-1}(\tau, \lambda)=F^{1}(\tau, \lambda)^{\perp} .
\end{aligned}
$$

Here $F(\tau, \lambda) \in D$ if, and only if,

$$
\operatorname{det}(\operatorname{Im} \tau)<0, \quad-i\langle\omega, \bar{\omega}\rangle>0 \quad \text { for } 0 \neq \omega \in F^{1}(\tau, \lambda) .
$$

Let $\sigma$ be a nilpotent cone, and let $\Gamma$ be a subgroup of $G_{\mathbb{Z}}$ such that

$$
\text { there exists } \gamma \in \Gamma \text { which satisfies } \sigma=\mathbb{R}_{\geq 0} \log (\gamma) \text {. }
$$

We write $\Gamma(\sigma)^{\mathrm{gp}}=\exp \left(\sigma_{\mathbb{R}}\right) \cap \Gamma$. The topology of $\Gamma(\sigma)^{\mathrm{gp}} \backslash D_{\sigma}$ is the quotient topology via the map $E_{\sigma} \rightarrow \Gamma(\sigma)^{\mathrm{gp}} \backslash D_{\sigma}([\mathrm{KU}, 3.4 .2])$. Here $E_{\sigma}$ is the subset of $\check{D} \times \mathbb{C}$ such that

$$
(F, z) \in E_{\sigma} \Leftrightarrow \begin{cases}\left(\sigma, \exp \left(\sigma_{\mathbb{C}}\right) F\right) \text { is a nilpotent orbit } & \text { if } z=0 \\ \exp (\ell(z) N) F \in D & \text { if } z \neq 0\end{cases}
$$

where $N$ is the generator of $\exp (\sigma) \cap \Gamma$ and $\ell(z)=\log z / 2 \pi i$ (we may assume that $\ell(z)$ is the one-valued function by taking a branch of log). The map $E_{\sigma} \rightarrow \Gamma(\sigma)^{\mathrm{gp}} \backslash D_{\sigma}$ is given by

$$
(F, z) \mapsto \begin{cases}\left(\sigma, \exp \left(\sigma_{\mathbb{C}}\right) F\right) & \text { if } z=0 \\ \exp (\ell(z) N) F \quad\left(\bmod \Gamma(\sigma)^{\mathrm{gp}}\right) & \text { if } z \neq 0 .\end{cases}
$$

The topology of $E_{\sigma}$ is the strong topology in $\check{D} \times \mathbb{C}$. For a fan $\Sigma$ and a subgroup $\Gamma$ of $G_{\mathbb{Z}}$ which is compatible with $\Sigma$, the topology of $\Gamma \backslash D_{\Sigma}$ is the strongest topology such that $\Gamma(\sigma)^{\mathrm{gp}} \backslash D_{\sigma} \rightarrow \Gamma \backslash D_{\Sigma}$ is continuous for all $\sigma \in \Sigma$.

We review the definition of the strong topology briefly. For an analytic space $Y$ and a subset $X$, a subset $U$ of $X$ is open in the strong topology in $Y$ if $f^{-1}(U)$ is 
open for any analytic space $Z$ and any analytic map $f: Z \rightarrow Y$ such that $f(Z) \subset X$. The following example is typical:

ExAmple 3.1 ([KU, 3.1.3]). Let $Y=\mathbb{C}^{2}$, and let $X=Y-\{0\} \times \mathbb{C}^{*}$. The strong topology on $X$ in $Y$ does not coincide with the topology as a subspace of $Y$ around the origin. We put

$$
\begin{aligned}
& U_{n}\left(\delta_{n}\right)=\left\{\left.\left(z_{1}, z_{2}\right) \in \Delta_{\delta_{n}}^{2}|| z_{1}\right|^{n}<\left|z_{2}\right|, z_{2} \neq 0\right\} \\
& U(\delta)=\bigcup_{n} U_{n}\left(\delta_{n}\right) \cup\{(0,0)\}
\end{aligned}
$$

where $\Delta_{\delta_{n}}$ is the $\delta_{n}$-open disk with $\delta_{n}>0$ and $\delta=\left\{\delta_{n}\right\}_{n}$. Then $U(\delta) \subset X$ is an open neighborhood of the origin and $U(\delta)$, where $\delta$ runs over all sequences in $\mathbb{R}_{>0}$, form a fundamental system of neighborhoods of the origin.

3.2. Boundaries for the type I. Let $\sigma=\mathbb{R}_{\geq 0} N$ be the type I nilpotent element with $N\left(e_{3}\right)=e_{1}$ and $N\left(e_{j}\right)=0$ for $j=1,2,4$. Here the LMHS of this type is described as the following diagram:

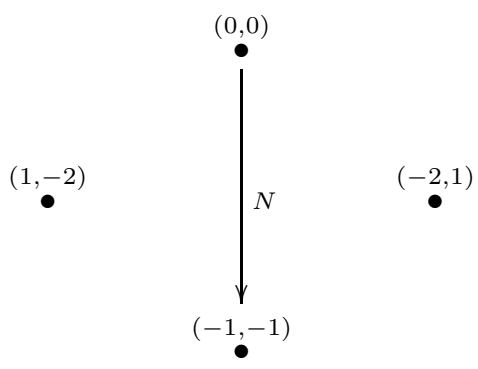

Then this nilpotent cone is even-type. We define a fan

$$
\Sigma_{\mathrm{ev}}=\left\{\operatorname{Ad}(g) \sigma \mid g \in G_{\mathbb{Z}}\right\} .
$$

Then $\Sigma_{\text {ev }}$ is the fan of all nilpotent cones of even-type by [KU, §12.3].

We write

$$
\xi_{0}(w)=\left(\begin{array}{c}
0 \\
w \\
1 \\
0
\end{array}\right), \quad \xi_{1}(v, w)=\left(\begin{array}{c}
w \\
v \\
0 \\
1
\end{array}\right)
$$

and define a filtration $F(v, w) \in \check{D}$ by

$$
F^{1}(v, w)=\operatorname{span}_{\mathbb{C}}\left\{\xi_{1}(v, w)\right\}, \quad F^{0}(v, w)=\operatorname{span}_{\mathbb{C}}\left\{\xi_{1}(v, w), \xi_{0}(w)\right\} .
$$

Then

$$
\mathbf{B}(\sigma)=\{(\sigma, \exp (\mathbb{C} N) F(v, w)) \mid \operatorname{Im} v<0, w \in \mathbb{C}\} .
$$

Let $F=F(v, w)$ with $\operatorname{Im} v<0$. Then the $(0,0)$-component of the Deligne decomposition for $(W(N), F)$ is

$$
F^{0} \cap W_{0}(N) \cap\left(\bar{F}^{0} \cap W_{0}(N)+\bar{F}^{-1} \cap W_{-2}(N)\right)=F^{0} \cap\left(\bar{F}^{0}+N \bar{F}^{0}\right),
$$


which is generated by

$$
e=\xi_{0}(w)-\gamma \xi_{1}(v, w)=\left(\begin{array}{c}
-\gamma w \\
\operatorname{Re} w-\gamma \operatorname{Re} v \\
1 \\
-\gamma
\end{array}\right), \quad \text { where } \gamma=\frac{\operatorname{Im} w}{\operatorname{Im} v}
$$

The $\mathbb{R}$-split mixed Hodge structure $(W(N), \hat{F})$ associated to $(W(N), F)$ is given by

$$
\hat{F}=\exp (\gamma i \operatorname{Im} w N) F .
$$

In fact, the $(0,0)$-component is generated by

$$
\hat{e}=\exp (\gamma i \operatorname{Im} w N) e=\left(\begin{array}{c}
-\gamma \operatorname{Re} w \\
\operatorname{Re} w-\gamma \operatorname{Re} v \\
1 \\
-\gamma
\end{array}\right)
$$

Then for the Hodge decomposition with respect to $\exp (i N) \hat{F}$,

$$
u_{1}:=\xi_{1}(v, w) \in H^{1,-2}, \quad u_{0}:=\exp (i N) \hat{e} \in H^{0,-1} .
$$

Here $H^{\mathrm{ev}}$ is generated by $u_{0}$ and $\bar{u}_{1}$, and by $(2.5)$

$$
\exp (X) u_{0}=2 i N \hat{e}=2 i e_{1}, \quad \exp (X) u_{1}=u_{1} .
$$

Then

$$
p^{\mathrm{ev}}\left(\sigma, \exp \left(\sigma_{\mathbb{C}}\right) F\right)=\exp (X) H^{\mathrm{ev}}=\operatorname{span}_{\mathbb{C}}\left\{e_{1}, \bar{v} e_{2}+e_{4}\right\},
$$

which is contained in $\mathbf{B}_{S}(\sigma)$. Moreover, the map $p^{\mathrm{ev}}: \mathbf{B}(\sigma) \rightarrow \mathbf{B}_{S}(\sigma)$ is surjective.

Now $\tilde{F}$ is given by $\tilde{F}^{0}=\operatorname{span}_{\mathbb{C}}\left\{\overline{\xi_{1}(v, w)}, \hat{e}\right\}$. By Proposition 2.10 , for $\operatorname{Im} z>0$

$$
\tilde{p}^{\operatorname{ev}}(\exp (z N) \hat{F})=\exp (z N) \tilde{F}=\left(\begin{array}{cc}
z-\gamma i \operatorname{Im} w & \bar{w} \\
\bar{w} & \bar{v}
\end{array}\right) .
$$

Since $\exp \left(\sigma_{\mathbb{C}}\right) F=\exp \left(\sigma_{\mathbb{C}}\right) \hat{F}$, we have

$$
\tilde{p}^{\mathrm{ev}}\left(\sigma, \exp \left(\sigma_{\mathbb{C}}\right) F\right)=\left(\sigma, \exp \left(\sigma_{\mathbb{C}}\right) \tilde{F}\right) .
$$

Proposition 3.2. Let $\Gamma$ be a subgroup of $G_{\mathbb{Z}}$ which is compatible with $\Gamma$ and satisfies the condition (3.3) for any $\sigma \in \Sigma_{\mathrm{ev}}$. Then $\tilde{p}^{\mathrm{ev}}: \Gamma \backslash D_{\Sigma_{\mathrm{ev}}} \rightarrow \Gamma \backslash \mathscr{H}_{\Sigma_{\mathrm{ev}}}$ is continuous.

Proof. It is sufficient to show continuity around the boundary point $\left(\sigma, \exp \left(\sigma_{\mathbb{C}}\right) F(v, w)\right)$ in $\Gamma \backslash D_{\Sigma_{\mathrm{ev}}}$. We write

$$
F=F(v, w), \quad \xi_{0}=\xi_{0}(w), \quad \xi_{1}=\xi_{1}(v, w),
$$

and we assume $F=\hat{F}$, i.e. $\operatorname{Im} w=0$ (it is similar to show continuity for $F \neq \hat{F}$, and we omit the proof of it). A neighborhood of $\left(\sigma, \exp \left(\sigma_{\mathbb{C}}\right) \tilde{F}\right)$ in $\Gamma \backslash \mathscr{H}_{\Sigma_{\text {ev }}}$ is written as

$$
\begin{aligned}
& \left\{\exp \left(\ell\left(z_{4}^{\prime}\right) N\right) \tilde{F}\left(z^{\prime}\right) \mid z^{\prime} \in \Delta_{\varepsilon}^{4}, z_{4}^{\prime} \neq 0\right\} \\
& \sqcup\left\{\exp \left(\sigma_{\mathbb{C}}\right) \tilde{F}\left(z^{\prime}\right) \mid z^{\prime} \in \Delta_{\varepsilon}^{4}, z_{4}^{\prime}=0\right\}
\end{aligned}
$$


for sufficiently small $\varepsilon>0$ where

$$
\tilde{F}\left(z^{\prime}\right)=\tilde{F}+\left(\begin{array}{ll}
z_{1}^{\prime} & z_{2}^{\prime} \\
z_{2}^{\prime} & z_{3}^{\prime}
\end{array}\right)
$$

We describe neighborhoods of $\left(\sigma, \exp \left(\sigma_{\mathbb{C}}\right) F\right)$ of $\Gamma \backslash D_{\Sigma_{\mathrm{ev}}}$, and show that there is a small neighborhood whose image through $\tilde{p}^{\text {ev }}$ is contained in the above neighborhood $(3.5)$.

A neighborhood of the boundary point $\left(\sigma, \exp \left(\sigma_{\mathbb{C}}\right) F\right)$ in $\Gamma \backslash D_{\Sigma_{\text {ev }}}$ is given by $E_{\sigma}$ and the map $\phi: E_{\sigma} \rightarrow \Gamma \backslash D_{\Sigma_{\text {ev }}}$. It is sufficient to show $\tilde{p}^{\text {ev }} \circ \phi$ is continuous. We describe a neighborhood of $(F, 0) \in E_{\sigma} \subset \check{D} \times \mathbb{C}$. Let $\Delta$ be a small open disk. By (3.1) an open neighborhood of $F$ in $\check{D}$ is given by

$$
\begin{aligned}
& \Delta^{4} \hookrightarrow \check{D} ; \quad\left(z_{1}, \ldots, z_{4}\right) \mapsto F(z) \\
& \text { where } F^{1}(z)=\operatorname{span}_{\mathbb{C}}\left\{\xi_{1}+\theta_{1}(z)+z_{4}\left(\xi_{0}+\theta_{0}(z)\right)\right\}, \\
& F^{0}(z)=\operatorname{span}_{\mathbb{C}}\left\{\xi_{1}+\theta_{1}(z), \xi_{0}+\theta_{0}(z)\right\}
\end{aligned}
$$

where

$$
\theta_{0}(z)=\left(\begin{array}{c}
z_{1} \\
z_{2} \\
0 \\
0
\end{array}\right), \quad \theta_{1}(z)=\left(\begin{array}{c}
z_{2} \\
z_{3} \\
0 \\
0
\end{array}\right)
$$

Then we have an open neighborhood $\Delta^{5} \hookrightarrow \check{D} \times \mathbb{C}$ by $\left(z_{1}, \ldots, z_{5}\right) \mapsto\left(F\left(z_{1}, \ldots, z_{4}\right), z_{5}\right)$. Here

$$
\left(F\left(z_{1}, \ldots, z_{4}\right), z_{5}\right) \in E_{\sigma} \Leftrightarrow \begin{cases}z_{4}=0 & \text { if } z_{5}=0 \\ \exp \left(\ell\left(z_{5}\right) N\right) F\left(z_{1}, \ldots, z_{4}\right) \in D & \text { if } z_{5} \neq 0\end{cases}
$$

For $z_{5} \neq 0$, we write

$$
\begin{aligned}
& \eta_{0}(z)=e^{\ell\left(z_{5}\right) N}\left(\xi_{0}+\theta_{0}(z)\right)=e^{\ell\left(z_{5}\right) N} \xi_{0}+\theta_{0}(z), \\
& \eta_{1}(z)=e^{\ell\left(z_{5}\right) N}\left(\xi_{1}+\theta_{1}(z)\right)+z_{4} \eta_{0}(z)=\xi_{1}+\theta_{1}(z)+z_{4} \eta_{0}(z) .
\end{aligned}
$$

Then

$$
e^{\ell\left(z_{5}\right) N} F^{1}(z)=\operatorname{span}_{\mathbb{C}}\left\{\eta_{1}(z)\right\}, \quad e^{\ell\left(z_{5}\right) N} F^{0}(z)=\operatorname{span}_{\mathbb{C}}\left\{\eta_{1}(z), \eta_{0}(z)\right\}
$$

If $z_{1}, \ldots, z_{5} \rightarrow 0$ provided that $\left|z_{4}\right| \log \left|z_{5}\right| \rightarrow 0$, we then have the convergences

$$
\begin{aligned}
& \eta_{1}(z) \rightarrow \xi_{1}, \quad \eta_{0}(z)-e^{\ell\left(z_{5}\right) N} \xi_{0} \rightarrow 0 \\
& \left\langle\overline{\eta_{1}(z)}, \eta_{1}(z)\right\rangle \rightarrow\left\langle\overline{\xi_{1}}, \xi_{1}\right\rangle, \quad\left\langle\overline{\eta_{1}(z)}, \eta_{0}(z)\right\rangle-\left\langle\overline{\xi_{1}}, e^{\ell\left(z_{5}\right) N} \xi_{0}\right\rangle \rightarrow 0 .
\end{aligned}
$$

Here

$$
e^{\ell\left(z_{5}\right) N} F^{1}=\operatorname{span}_{\mathbb{C}}\left\{\xi_{1}\right\}, \quad e^{\ell\left(z_{5}\right) N} F^{0}=\operatorname{span}_{\mathbb{C}}\left\{\xi_{1}, e^{\ell\left(z_{5}\right) N} \xi_{0}\right\}
$$

Then

$$
e^{\ell\left(z_{5}\right) N} F(z)-e^{\ell\left(z_{5}\right) N} F \rightarrow 0
$$


if $z_{1}, \ldots, z_{5} \rightarrow 0$ provided that $\left|z_{4}\right| \log \left|z_{5}\right| \rightarrow 0$. Therefore, by the conditions (3.2) of $D$,

$$
U_{n}\left(\delta_{n}\right)=\left\{\begin{array}{l|l}
\left(z_{1}, \ldots, z_{5}\right) \in \Delta^{5} \mid \begin{array}{l}
\left|z_{4}\right|^{n}<\left|z_{5}\right|, \\
\left|z_{4}\right|,\left|z_{5}\right|<\delta_{n}, z_{5} \neq 0
\end{array}
\end{array}\right\} \subset E_{\sigma}
$$

if $\delta_{n}$ is sufficiently small for $n \geq 1$. Here $U_{n}\left(\delta_{n}\right)$ is a small neighborhood of $\left\{e^{\ell\left(z_{5}\right) N} F|| z_{5} \mid<\delta_{n}\right\}$. By Example 3.1 and the definition of strong topology,

$$
U(\delta)=\left(\bigcup_{n} U_{n}\left(\delta_{n}\right)\right) \sqcup\left\{z \in \Delta^{5} \mid z_{4}=z_{5}=0\right\}
$$

is an open neighborhood of $(0, F)$ in the strong topology of $E_{\sigma}$ taking $\delta=\left\{\delta_{n}\right\}$ and $\Delta$ sufficiently small.

Next, we show $p^{\text {ev }}\left(e^{\ell\left(z_{5}\right) N} F(z)\right)$ approaches $e^{\ell\left(z_{5}\right) N} \tilde{F}$ if $z_{1}, \ldots, z_{5} \rightarrow 0$ provided that $z \in U_{n}\left(\delta_{n}\right)$, i.e. $\left|z_{4}\right| \log \left|z_{5}\right| \rightarrow 0$. For the Hodge decomposition for $e^{\ell\left(z_{5}\right) N} F(z)$ with $\left(z_{1}, \ldots, z_{5}\right) \in U_{n}\left(\delta_{n}\right)$, the $(1,-2)$ component is generated by $\eta_{1}(z)$ and the $(0,-1)$ component is generated by

$$
\alpha(z)=\left\langle\overline{\eta_{1}(z)}, \eta_{0}(z)\right\rangle \eta_{1}(z)-\left\langle\overline{\eta_{1}(z)}, \eta_{1}(z)\right\rangle \eta_{0}(z)
$$

since $\overline{\eta_{1}(z)} \perp \alpha(z)$. Then

$$
p^{\mathrm{ev}}\left(e^{\ell\left(z_{5}\right) N} F(z)\right)=\operatorname{span}_{\mathbb{C}}\left\{\overline{\eta_{1}(z)}, \alpha(z)\right\}
$$

We write

$$
\beta\left(z_{5}\right)=\left\langle\overline{\xi_{1}}, e^{\ell\left(z_{5}\right) N} \xi_{0}\right\rangle \xi_{1}-\left\langle\overline{\xi_{1}}, \xi_{1}\right\rangle e^{\ell\left(z_{5}\right)} \xi_{0},
$$

which is in the $(0,-1)$ component of the Hodge decomposition of $e^{\ell\left(z_{5}\right) N} F$. Then, by Proposition 2.10,

$$
\operatorname{span}_{\mathbb{C}}\left\{\overline{\xi_{1}}, \beta\left(z_{5}\right)\right\}=p^{\mathrm{ev}}\left(e^{\ell\left(z_{5}\right) N} F\right)=e^{\ell\left(z_{5}\right) N} \tilde{F} .
$$

By the convergence (3.6) we have

$$
\eta_{1}(z) \rightarrow \xi_{1}, \quad \alpha(z)-\beta\left(z_{5}\right) \rightarrow 0
$$

if $z_{1}, \ldots, z_{5} \rightarrow 0$ provided that $z \in U_{n}\left(\delta_{n}\right)$. Moreover we have

$$
p^{\mathrm{ev}}\left(e^{\ell\left(z_{5}\right) N} F(z)\right)-e^{\ell\left(z_{5}\right) N} \tilde{F} \rightarrow 0
$$

in the upper half space. Then $U(\delta)$ is contained in the neighborhood (3.5) if $\delta_{n}$ is sufficiently small.

Corollary 3.3. $p^{\text {ev }}$ is continuous.

REMARK 3.4. The $G_{\mathbb{Z}}\left(\mathbf{B}_{S}(\sigma)\right)$-admissible polyhedral decomposition ([CCK, Definition 6.1]) is the fan $\{\sigma,\{0\}\}$. Then for a $\Gamma$-admissible decomposition $\Sigma_{\text {tor }}$ ([CCK, Definition 6.2]), we have an injection $\Sigma_{\mathrm{ev}} \hookrightarrow \Sigma_{\text {tor }}$. Therefore we have the following commutative diagram:

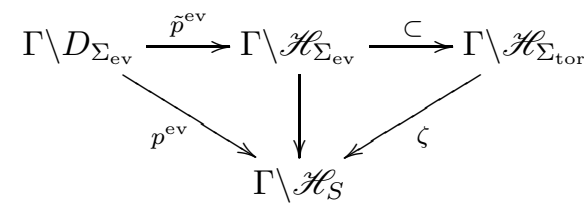


3.3. Boundaries for the type II. Let $\sigma_{m}=\mathbb{R}_{\geq 0} N_{m}$ be the type II nilpotent cone with $N_{m} e_{3}=-e_{1}$ and $N_{m} e_{4} \mapsto-m e_{2}$ where $m$ is a square-free positive integer. The LMHS of this type is described as the following diagram:
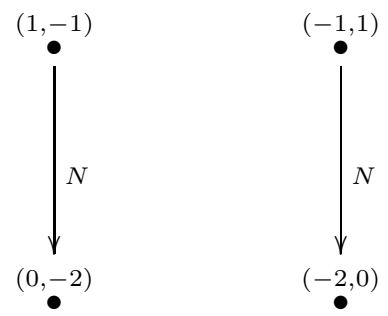

Then this nilpotent cone is odd-type. We define

$$
\Sigma_{\mathrm{od}}=\left\{\operatorname{Ad}(g) \sigma_{m} \mid g \in G_{\mathbb{Z}}, m: \text { square-free positive integer }\right\} .
$$

Then $\Sigma_{\text {od }}$ is the fan of all nilpotent cones of odd-type by [KU, $\left.\S 12.3\right]$.

Let $N=N_{m}$, and let $\sigma=\sigma_{m}$. We write

$$
\xi_{0}^{ \pm}=\left(\begin{array}{c}
-1 \\
\pm i \sqrt{m} \\
0 \\
0
\end{array}\right), \quad \xi_{1}^{ \pm}(w)=\left(\begin{array}{c}
0 \\
w \\
\pm i \sqrt{m} \\
1
\end{array}\right)
$$

Then for $z \in \mathbb{C}$

$$
N \xi_{1}^{ \pm}(w)= \pm i \sqrt{m} \xi_{0}^{ \pm}, \quad \xi_{1}^{ \pm}(w) \pm z i \sqrt{m} \xi_{0}^{ \pm}=\exp (z N) \xi_{1}^{ \pm}(w) .
$$

We define a filtration $F_{ \pm}(w) \in \check{D}$ by

$$
F_{ \pm}^{1}(w)=\operatorname{span}_{\mathbb{C}}\left\{\xi_{1}^{ \pm}(w)\right\}, \quad F_{ \pm}^{0}(w)=\operatorname{span}_{\mathbb{C}}\left\{\xi_{1}^{ \pm}(w), \xi_{0}^{ \pm}\right\}
$$

Then

$$
\mathbf{B}(\sigma)=\left\{\left(\sigma, \exp (\mathbb{C} N) F_{+}(w)\right) \mid w \in \mathbb{C}\right\} \sqcup\left\{\left(\sigma, \exp (\mathbb{C} N) F_{-}(w)\right) \mid w \in \mathbb{C}\right\} .
$$

Let $F=F_{ \pm}(w)$ and let $\sigma=\mathbb{R}_{\geq 0} N$. Then the $(1,-1)$ component of the Deligne decomposition of $(W(N), F)$ is generated by $\xi_{1}^{ \pm}(w)$, and the $(-1,1)$ component is

$$
F^{-1} \cap\left(\bar{F}^{1}+\bar{F}^{0} \cap W_{-2}(N)\right)=F^{-1} \cap\left(\bar{F}^{1}+N \bar{F}^{1}\right),
$$

which is generated by

$$
\begin{aligned}
\omega & =\left\langle\xi_{1}^{ \pm}(w), \overline{\xi_{0}^{ \pm}}\right\rangle \overline{\xi_{1}^{ \pm}(w)}-\left\langle\xi_{1}^{ \pm}(w), \overline{\xi_{1}^{ \pm}(w)}\right\rangle \overline{\xi_{0}^{ \pm}} \\
& =\mp 2 i \sqrt{m} \overline{\xi_{1}^{ \pm}(w)}+2 i \operatorname{Im} w \overline{\xi_{0}^{ \pm}} \\
& =\mp 2 i \sqrt{m} \exp \left(-\frac{\operatorname{Im} w}{m} i N\right) \overline{\xi_{1}^{ \pm}(w)} .
\end{aligned}
$$

The $\mathbb{R}$-split MHS $(W(N), \hat{F})$ associated to $(W(N), F)$ is given by

$$
\hat{F}=\exp \left(\frac{\operatorname{Im} w}{2 m} i N\right) F \text {. }
$$


In fact, the $(1,-1)$ component is generated by

$$
\hat{\xi}=\exp \left(\frac{\operatorname{Im} w}{2 m} i N\right) \xi_{1}^{ \pm}(w)
$$

and the $(-1,1)$ component is generated by

$$
\hat{\omega}=\exp \left(\frac{\operatorname{Im} w}{2 m} i N\right) \omega=\mp 2 i \sqrt{m} \exp \left(-\frac{\operatorname{Im} w}{2 m} i N\right) \overline{\xi_{1}^{ \pm}(w)} .
$$

Then the Hodge decomposition with respect to $\exp (i N) \hat{F} \in D$ is given by

$$
u_{1}=\exp (i N) \hat{\xi} \in H^{1,-2}, \quad \bar{u}_{0}=\exp (i N) \hat{\omega} \in H^{-1,0} .
$$

Here $H^{\text {od }}$ is generated by $u_{1}$ and $\bar{u}_{0}$, and by $(2.5)$

$$
\exp (X) u_{1}=2 i N \hat{\xi}, \quad \exp (X) \bar{u}_{0}=2 i N \hat{\omega} .
$$

Then

$$
p^{\text {od }}\left(\sigma, \exp \left(\sigma_{\mathbb{C}}\right) F\right)=\exp (X) H^{\text {od }}=\operatorname{span}_{\mathbb{C}}\left\{e_{1}, e_{2}\right\}
$$

which is contained in $\overline{\mathbf{B}_{S}(\sigma)}$. Moreover, the map $p^{\text {od }}: \mathbf{B}(\sigma) \rightarrow \overline{\mathbf{B}_{S}(\sigma)}$ is surjective.

Now $\tilde{F} \in \check{\mathscr{H}}$ is given by $\tilde{F}^{0}=\operatorname{span}_{\mathbb{C}}\{\hat{\xi}, \hat{\omega}\}$. By Proposition 2.10 , for $\operatorname{Im} z>0$

$$
p^{\text {od }}(\exp (z N) F)=\exp (z N) \tilde{F}=\left(\begin{array}{cc}
-z & \pm \frac{\operatorname{Im} w}{2 \sqrt{m}} \\
\pm \frac{\operatorname{Im} w}{2 \sqrt{m}} & \operatorname{Re} w-m z
\end{array}\right)
$$

Since $\exp \left(\sigma_{\mathbb{C}}\right) F=\exp \left(\sigma_{\mathbb{C}}\right) \hat{F}$, we have

$$
\tilde{p}^{\text {od }}\left(\sigma, \exp \left(\sigma_{\mathbb{C}}\right) F\right)=\left(\sigma, \exp \left(\sigma_{\mathbb{C}}\right) \tilde{F}\right) .
$$

Here $\tilde{p}^{\text {od }}$ is not surjective.

Proposition 3.5. Let $\Gamma$ be a subgroup of $G_{\mathbb{Z}}$ which is compatible with $\Gamma$ and satisfies the condition (3.3) for any $\sigma \in \Sigma_{\mathrm{od}}$. Then $\tilde{p}^{\text {od }}: \Gamma \backslash D_{\Sigma_{\mathrm{od}}} \rightarrow{\overline{\Gamma \backslash \mathscr{H}_{\Sigma_{\mathrm{od}}}}}_{\text {is }}$ continuous.

Proof. As in the proof of Proposition 3.2, it is sufficient to show continuity around the boundary point $\left(\sigma, \exp \left(\sigma_{\mathbb{C}}\right) F_{ \pm}(w)\right)$ in $\Gamma \backslash D_{\Sigma_{\text {od }}}$. We write

$$
F=\hat{F}_{ \pm}(w), \quad \xi_{0}=\xi_{0}^{ \pm}, \quad \xi_{1}=\xi_{1}^{ \pm}(w),
$$

and we assume $F=\hat{F}$, i.e. $\operatorname{Im} w=0$ (it is similar to show continuity for $F \neq \hat{F}$ and we omit the proof of it). We describe neighborhoods of $\left(\sigma, \exp \left(\sigma_{\mathbb{C}}\right) F\right)$ of $\Gamma \backslash D_{\Sigma_{\text {od }}}$, and show the continuity.

A neighborhood of a boundary point $\left(\sigma, \exp \left(\sigma_{\mathbb{C}}\right) F\right)$ in $\Gamma \backslash D_{\sigma}$ is given by $E_{\sigma}$ and the map $\phi: E_{\sigma} \rightarrow \Gamma \backslash D_{\Sigma_{\text {od }}}$. It is sufficient to show $\tilde{p}^{\text {od }} \circ \phi$ is continuous. We describe a neighborhood of $(F, 0) \in E_{\sigma} \subset \check{D} \times \mathbb{C}$. An open neighborhood of $F$ in $\check{D}$ is given by

$$
\begin{aligned}
& \Delta^{4} \hookrightarrow \check{D} ; \quad\left(z_{1}, \ldots, z_{4}\right) \mapsto F(z) \\
& \text { where } F^{1}(z)=\operatorname{span}_{\mathbb{C}}\left\{\xi_{1}+\theta_{1}(z)+z_{4}\left(\xi_{0}+\theta_{0}(z)\right)\right\}, \\
& F^{0}(z)=\operatorname{span}_{\mathbb{C}}\left\{\xi_{1}+\theta_{1}(z), \xi_{0}+\theta_{0}(z)\right\},
\end{aligned}
$$


and

$$
\theta_{0}(z)=\left(\begin{array}{c}
0 \\
z_{2} \\
z_{1} \\
0
\end{array}\right), \quad \theta_{1}(z)=\left(\begin{array}{c}
0 \\
z_{3} \\
z_{2} \\
0
\end{array}\right)
$$

Then we have an open neighborhood $\Delta^{5} \hookrightarrow \check{D} \times \mathbb{C}$ by $\left(z_{1}, \ldots, z_{5}\right) \mapsto\left(F\left(z_{1}, \ldots, z_{4}\right), z_{5}\right)$. Here

$$
\left(F\left(z_{1}, \ldots, z_{4}\right), z_{5}\right) \in E_{\sigma} \Leftrightarrow \begin{cases}z_{1}=z_{2}=0 & \text { if } z_{5}=0 \\ \exp \left(\ell\left(z_{5}\right) N\right) F\left(z_{1}, \ldots, z_{4}\right) \in D & \text { if } z_{5} \neq 0\end{cases}
$$

For $z_{5} \neq 0$, we write

$$
\begin{aligned}
& \eta_{0}(z)=e^{\ell\left(z_{5}\right) N}\left(\xi_{0}+\theta_{0}(z)\right)=\xi_{0}+e^{\ell\left(z_{5}\right) N} \theta_{0}(z) \\
& \eta_{1}(z)=e^{\ell\left(z_{5}\right) N}\left(\xi_{1}+\theta_{1}(z)\right)+z_{4} \eta_{0}(z) .
\end{aligned}
$$

Then

$$
e^{\ell\left(z_{5}\right) N} F^{1}(z)=\operatorname{span}_{\mathbb{C}}\left\{\eta_{1}(z)\right\}, \quad e^{\ell\left(z_{5}\right) N} F^{0}(z)=\operatorname{span}_{\mathbb{C}}\left\{\eta_{1}(z), \eta_{0}(z)\right\} .
$$

If $z_{1}, \ldots, z_{5} \rightarrow 0$ provided that $\left|z_{1}\right| \log \left|z_{5}\right| \rightarrow 0$ and $\left|z_{2}\right| \log \left|z_{5}\right| \rightarrow 0$, then we have

$$
\begin{aligned}
& \eta_{1}(z)-e^{\ell\left(z_{5}\right) N} \xi_{1} \rightarrow 0, \quad \eta_{0}(z) \rightarrow \xi_{0}, \\
& \left\langle\overline{\eta_{1}(z)}, \eta_{1}(z)\right\rangle-\left\langle\overline{e^{\ell\left(z_{5}\right) N} \xi_{1}}, e^{\ell\left(z_{5}\right) N} \xi_{1}\right\rangle \rightarrow 0, \\
& \left.\left\langle\overline{\eta_{1}(z)}, \eta_{0}(z)\right\rangle \rightarrow \overline{\left\langle e^{\ell\left(z_{5}\right) N} \xi_{1}\right.}, \xi_{0}\right\rangle=\left\langle\overline{\xi_{1}}, \xi_{0}\right\rangle .
\end{aligned}
$$

Here

$$
e^{\ell\left(z_{5}\right) N} F^{1}=\operatorname{span}_{\mathbb{C}}\left\{e^{\ell\left(z_{5}\right) N} \xi_{1}\right\}, \quad e^{\ell\left(z_{5}\right) N} F^{0}=\operatorname{span}_{\mathbb{C}}\left\{e^{\ell\left(z_{5}\right) N} \xi_{1}, \xi_{0}\right\}
$$

Then

$$
e^{\ell\left(z_{5}\right) N} F(z)-e^{\ell\left(z_{5}\right) N} F \rightarrow 0
$$

if $z_{1}, \ldots, z_{5} \rightarrow 0$ provided that $\left|z_{1}\right| \log \left|z_{5}\right| \rightarrow 0$ and $\left|z_{2}\right| \log \left|z_{5}\right| \rightarrow 0$. Therefore, by the conditions (3.2) of $D$,

$$
U_{n, m}\left(\delta_{n, m}\right)=\left\{\begin{array}{l|l}
\left(z_{1}, \ldots, z_{5}\right) \in \Delta^{5} & \begin{array}{l}
\left|z_{1}\right|^{n}<\left|z_{5}\right|,\left|z_{2}\right|^{m}<\left|z_{5}\right| \\
\left|z_{1}\right|,\left|z_{2}\right|,\left|z_{5}\right|<\delta_{n, m}, z_{5} \neq 0
\end{array}
\end{array}\right\} \subset E_{\sigma}
$$

if $\delta_{n, m}$ and $\Delta$ is sufficiently small. By Example 3.1 and the definition of strong topology,

$$
U(\delta)=\left(\bigcup_{n, m} U_{n, m}\left(\delta_{n, m}\right)\right) \sqcup\left\{z \in \Delta^{5} \mid z_{1}=z_{2}=z_{5}=0\right\}
$$

is an open neighborhood of $(F, 0)$ in $E_{\sigma}$ for $\delta=\left\{\delta_{n, m}\right\}$.

As in the proof of the case for even-types, we can show that

$$
p^{\text {od }}\left(e^{\ell\left(z_{5}\right) N} F(z)\right)-e^{\ell\left(z_{5}\right) N} \tilde{F} \rightarrow 0
$$


if $z_{1}, \ldots, z_{5} \rightarrow 0$ provided that $z \in U_{n, m}\left(\delta_{n, m}\right)$. Then $\tilde{p}^{\text {od }} \circ \phi(U(\delta))$ is contained in the open neighborhood (3.5) if $\delta_{n, m}$ is sufficiently small.

COROLlary 3.6. $p^{\text {od }}$ is continuous.

REMARK 3.7. A $G_{\mathbb{Z}}\left(\mathbf{B}_{S}(\sigma)\right)$-admissible decomposition $\Sigma$ does not contain $\sigma$ in this case. Then we do not have a diagram like the one in Remark 3.4.

\section{REFERENCES}

[AmRT] A. Ash, D. Mumford, M. Rapoport, And Y. S. Tai, Smooth compactification of locally symmetric varieties, Math. Sci. Press, Brookline, 1975.

[CCK] J. A. Carlson, E. Cattani, and A. Kaplan, Mixed Hodge structures and compactification of Siegel's space, in Journées de géométrie algébrique d'Angers 1979 (A. Beauville, ed.), Sijthohoff \& Nordhoff, 1980, pp. 77-105.

[CK] E. Cattani and A. Kaplan, Polarized mixed Hodge structures and the local monodromy of a variation of Hodge structure, Invent. Math., 67:1 (1982), pp. 101-115.

[CKS] E. Cattani, A. Kaplan, and W. Schmid, Degeneration of Hodge structures, Ann. of Math., 123 (1986), pp. 457-535.

[CMP] J. Carlson, S. Müller-Stach, and C. Peters, Period mappings and period domains, Cambridge Studies in Advanced Mathematics, 85. Cambridge University Press, Cambridge, 2003.

[FHW] G. Fels, A. Huckleberry, and J. A. Wolf, Cycle Spaces of Flag Domains: A Complex Geometric Viewpoint, Progress in Mathematics, 245, Birkhauser Boston, Inc., 2006.

[G] P. GRiffiths, Periods of integrals on algebraic manifolds. I. Construction and properties of the modular varieties, Amer. J. Math., 90 (1968), pp. 568-626.

[GGK1] M. Green, P. Griffiths, and M. KerR, Néron models and boundary components for degenerations of Hodge structures of mirror quintic type, in "Curves and Abelian Varieties" (V. Alexeev, Ed.), Contemp. Math, 465 (2007), AMS, pp. 71-145.

[GGK2] M. Green, P. GRIffiths, And M. KerR, Mumford-Tate groups and domains: their geometry and arithmetic, Annals of Math. Studies, 183, Princeton University Press, 2012.

[H] T. Hayama, On the boundary of the moduli spaces of log Hodge structures, II: non-trivial torsors, Nagoya Math. J., 213 (2014), pp. 1-20.

[H2] T. Hayama, Kato-Usui partial compactifications over the toroidal compactifications of Siegel spaces, Contemp. Math. AMS, 608 (2014), pp. 143-155.

[KU] K. Kato and S. Usui, Classifying space of degenerating polarized Hodge structures, Annals of Mathematics Studies, 169, Princeton University Press, Princeton, NJ, 2009.

[KP] M. Kerr and G. Pearlstein, Boundary components of Mumford-Tate domains, preprint.

[N] Y. NAmiKaWA, Toroidal compactification of Siegel spaces, Lecture Notes in Math., 812, Springer-Verlag, 1980.

[S] W. Schmid, Variation of Hodge structure: the singularities of the period mapping, Invent. Math., 22 (1973), pp. 211-319. 
T. HAYAMA 